\title{
Functional limitations and worrying to lose employment among individuals with chronic conditions and disabilities during COVID-19: A hierarchical logistic regression model
}

\author{
Emre Umucu* \\ Department of Rehabilitation Sciences, Master of Rehabilitation Counseling Program, University of Texas at El \\ Paso, El Paso, TX, USA
}

Received 10 March 2020

Accepted 28 September 2020

\begin{abstract}
.
BACKGROUND: As an unexpected environmental factor causing significant economic, financial, and health challenges for everyone, it is no wonder that COVID-19 presents significant employment-related worries for people with chronic health conditions and disabilities.

OBJECTIVE: The purpose of this study was to explore whether higher levels of functional limitations are associated with worry about job loss for individuals with disabilities and chronic conditions.

METHODS: Data for this study were collected to understand the effects of COVID-19 on individuals with disabilities and chronic conditions. A total of 219 employed participants were extracted from a total of 269 individuals with disabilities and chronic conditions. The mean age among participants was $37.83(\mathrm{SD}=10.97)$.

RESULTS: Our hierarchical logistic regression analysis revealed that (a) demographic variables were not associated with worry about job loss in the first block, (b) perceived COVID-19 stress (AOR $=2.90, p<0.001$ ) was correlated with worry about job loss in the second block, and (c) functional limitations ( $\mathrm{AOR}=1.06, p<0.001$ ) were positively correlated with worry about job loss in the third block. Overall, the entire model containing all predictor variables in three blocks explained $23 \%$ (Nagelkerke $R^{2}$ ) of the variance in worry about job loss.

CONCLUSIONS: This study contributes to the disability and vocational rehabilitation literature by examining the relationship between functional limitations and worry to lose jobs in people with disabilities.
\end{abstract}

Keywords: Employment, COVID-19, functional limitations, anxiety

\section{Introduction}

There are more than one billion people with disabilities worldwide (Armitage \& Nellums, 2020). People with disabilities have a higher likelihood of

\footnotetext{
*Address for correspondence: Emre Umucu, Assistant Professor, Department of Rehabilitation Sciences, University of Texas at El Paso, 500 W University Ave, El Paso, Texas, 79968, USA. Tel.: +1915 747 7275; E-mail: eumucu@utep.edu.
}

(a) experiencing poverty, (b) facing higher healthcare needs and more barriers in accessing services, and (c) being excluded from education (Kuper \& Heydt, 2019). In addition, individuals with disabilities often face difficulties in obtaining and maintaining employment (Chan et al., 2010; Kuper \& Heydt, 2019; Potts, 2005), which could be considered a significant public health concern given that researchers (Chiu et al., 2013; Dutta et al., 2008; Kuper \& Heydt, 2019) have reported that employment plays an integral 
role in one's physical and psychological well-being. For example, Muller et al. (2017) reported that unemployed individuals with disabilities (i.e., fibromyalgia) had the highest number of secondary health conditions, the lowest levels of health related quality of life, the highest levels of depression, and the lowest levels of social support and life satisfaction when compared to full-time employed and part-time employed individuals with disabilities. Given that employment is associated with desirable health and well-being outcomes, it is important to examine factors affecting employment in people with disabilities.

Functional limitations may increase the risk of unemployment and underemployment among people with disabilities. One study examined absenteeism due to functional limitations in US workers (Vuong et al., 2015). Their findings revealed that absenteeism was significantly associated with functional limitations in people with chronic conditions (Vuong et al., 2015). In addition, their results revealed that employees with multiple chronic conditions had higher rates of absenteeism. Brucker et al. (2016) examined employment outcomes in individuals with disabilities by using sensory, functional, and activity limitation data. They reported that the presence of an activity limitation (e.g., self-care) was strongly related to reduced odds of employment in individuals with sensory or functional limitations (Brucker et al., 2016). Lee et al. (2019) examined whether functional limitations predicted work participation in head and neck cancer survivors. Their results revealed that, compared to participants who were unemployed, participants who were employed had lower levels of functional limitations in cognition, mobility, selfcare, getting along with people, daily life activities, and participation in society (Lee et al., 2019).

The relationship between functioning and employment has been examined thoroughly in the rehabilitation literature. There is, however, a lack of research examining whether functional limitations increase worry about loss of employment in individuals with disabilities. Lindesay et al. (2006) examined the associations among age, worry, mental disorders, and functional limitations in a community sample. They categorized worry items into five domains: relationships/family, financial/housing, work, health, and miscellaneous. They found that there was a negative association between worry and functional limitations and that this negative relationship was anomalous (Lindesay et al., 2006). The researchers speculated that "... this [relationship] is significant only for more severe degrees of functional limitation, so this may reflect the fact that more severely disabled individuals are relieved of the need to seek or hold down a job, and therefore the worry associated with this" (p.1632) (Lindesay et al., 2006).

With the current COVID-19 pandemic, individuals with disabilities and chronic conditions experience significant functional, financial, and overall health challenges (Armitage \& Nellums, 2020; Jalali et al., 2020; Umucu \& Lee, 2020; Umucu, Tansey, Brooks, \& Lee, 2020; World Health Organization [WHO], 2020). Due to COVID-19, the WHO (2020) reported some actions to reduce their potential viral exposure. For instance, one suggestion was that people with disabilities should work from home if possible. More specifically, the WHO reported that employers should consider flexible working arrangements allowing individuals with disabilities to telework and should provide technology for people with disabilities to complete their work from home (WHO, 2020). In case telework is not possible, employers may "consider allowing people with disabilities at high risk of severe symptoms to take leave (including paid leave) until the risk of infection is reduced" (WHO, 2020, p.14). Although these suggestions are very helpful for employers and communities, people with disabilities may feel high levels of stress and anxiety that they will lose their jobs given that not all employers are able to provide accommodations needed for work from home. In addition, individuals with higher levels of functional limitations may need more accommodations, which employers may have difficulty providing. The unavailability of these additional accommodations may increase stress and anxiety levels in individuals with disabilities and chronic illnesses.

\subsection{Purpose of the study}

As an unexpected environmental factor causing significant economic, financial, and health challenges for everyone, COVID-19 has been a significant risk factor for unemployment for both people with and without disabilities. To help people with disabilities during this pandemic, it is important to understand risk and protective factors in employment. This exploratory study therefore aimed to respond to the following research question: "Are higher levels of functional limitations significantly correlated with worry about job loss for individuals with disabilities and chronic conditions, after adjusting for demographic variables and perceived COVID-19 stress?" We hypothesized that higher levels of functional limitations 
would be associated with heightened worry about job loss among people with disabilities and chronic conditions during the COVID-19 pandemic.

\section{Method}

Data for this study were collected to understand the effects of COVID-19 on individuals with disabilities and chronic conditions (see Umucu \& Lee, 2020). We only extracted participants who were currently employed for this study $(N=219)$. A detailed description of the study procedures are reported in a previous research investigation we conducted (Umucu \& Lee, 2020).

\subsection{Participants}

A total of 219 employed participants were extracted from a total of 269 individuals with disabilities and chronic conditions. Regarding disability types, about $61 \%$ of participants reported physical disabilities, followed by psychiatric disabilities (19.2\%), learning disabilities (11\%), neurocognitive disabilities $(8.2 \%)$, sensory disabilities $(6.4 \%)$, and intellectual disabilities (2.3\%). The mean age among participants was $37.83(\mathrm{SD}=10.97)$. Among the participants, most $(59.8 \%)$ were male, white $(81.3 \%)$, and married $(57.5 \%)$. About $72 \%$ of participants had completed at least a bachelor's degree. Regarding employment, $84.9 \%$ of participants reported being paid employees and $15.1 \%$ were self-employed. About $64 \%$ of participants reported receiving disability benefits. Regarding workplace accommodations, about $66 \%$ of participants reported that their employers provided accommodation/s to increase their ability to work remotely. About $97.5 \%$ of participants reported following social distancing rules, and $93.2 \%$ of participants believed that social distancing is effective in reducing COVID-19 risk.

\subsection{Instrumentation}

\subsubsection{Demographic questionnaire}

We used a demographic questionnaire to gather data about participants' age, gender, race, marital status, and education. The demographic survey also had questions regarding COVID-19 (e.g., social distancing, accommodations, working remotely).

\subsubsection{Worry about job loss}

We used a single item to measure whether participants were worried to lose their jobs (i.e., "Are you worried to lose your job?"). This item is dichotomously coded as yes (1) vs. no (0).

\subsubsection{Perceived stress}

We used the adapted version of the Perceived Stress Questionnaire-8 (PSQ-8; Umucu et al., 2018), a shorter version of the Perceived Stress Questionnaire20 (PSQ-20; Fliege et al., 2005) to measure perceived COVID-19 stress. The scale was adapted by adding "Due to COVID-19" to the scale instruction (e.g., "Due to COVID-19, you feel tense?"). The PSQ-8 has a total of eight items rated on a 4-point Likert scale ranging from 1 (almost never) to 4 (usually). We calculated average COVID-19 related perceived stress scores ranging from 1 to 4 , with higher scores indicating higher levels of perceived stress. The internal consistency reliability coefficients ranged from 0.80 to 0.83 in previous studies (Umucu et al., 2018; Umucu \& Lee, 2020). In the current study, the internal consistency reliability coefficient was found to be 0.82 .

\subsubsection{Functional limitations}

We used the World Health Organization Disability Assessment Schedule II 12-item version (WHO DAS-2.0; Üstün et al., 2010) to measure functional limitations. Each item (e.g., In the last 30 days, how much difficulty did you have in: "Standing for long periods such as 30 minutes?") is rated on a 5-point Likert scale ranging from 1 (none) to 5 (extreme or cannot do). The total functional limitations score ranges from 12 to 60, with higher scores indicating greater functional limitations. The internal consistency reliability coefficient for the WHODAS 2.0 was 0.91 in a previous study (Umucu et al., 2020). In the current study, the internal consistency reliability coefficient was found to be 0.95 .

\subsection{Data analysis}

We utilized descriptive statistics to report participants' demographic characteristics. We conducted a correlational analysis to examine the relationships among variables. Finally, we conducted a hierarchical logistic regression to examine whether higher scores in functional limitations were associated with worry about job loss $(0=$ no, $1=$ yes $)$ after controlling for demographic variables and stress among people with disabilities and chronic conditions. In step 1, we entered demographic covariates (i.e., age, gen- 
$\operatorname{der}[1=$ male $]$, race $[1=$ white $]$ ); in step 2 we entered perceived COVID-19 stress; and step 3 we entered functional limitations. In this study, we considered demographic variables and perceived COVID-19 stress as covariates. All statistics were conducted with SPSS 26.0.

\section{Results}

\subsection{Descriptive statistics and correlational analysis}

The mean scores for stress and functional limitations were $2.46(\mathrm{SD}=0.67)$ and $29.49(\mathrm{SD}=12.16)$, respectively. A total of 124 participants reported that they were not worried about losing their jobs, and 95 participants reported that they were worried about losing their jobs. Our point-biserial correlation analysis results revealed that worry about job loss was positively associated with functional limitations $\left(r_{p . b}=0.36, p<0.001\right)$ and perceived COVID-19 stress $\left(r_{p . b}=0.31, p<0.001\right)$. The Pearson-correlation analysis results revealed that perceived COVID-19 stress was positively associated with functional limitations $(r=0.39, p<0.001)$.

\subsection{Hierarchical logistic regression analysis}

We entered demographic variables (i.e., age, gender, race) in the first block, followed by perceived COVID-19 stress in the second block, with functional limitations in the third block. The first block with demographic variables only was not significantly associated with worry about job loss $\left(\chi^{2}=1.088\right.$, $d f=3, p=0.78)$ with the Nagelkerke $R^{2}=0.007$.

The second block with demographic variables and perceived COVID-19 stress was significantly associated with worry about job loss $\left(\chi^{2}=23.815\right.$, $d f=4, p<0.000$ ) with the Nagelkerke $R^{2}=0.14$. By examining specific variables in the second block, perceived COVID-19 stress was significantly associated with worry about job loss (AOR $=2.90$, CI $95 \%$ [1.81-4.66], $p<0.000)$.

The third block with demographic variables, perceived COVID-19 stress, and functional limitations was associated with worry about job loss $\left(\chi^{2}=40.74\right.$, $d f=5, p=0.000$ ) with the Nagelkerke $R^{2}=0.23$. By examining specific variables in the third block, we found that functional limitation scores were significantly associated with worry about job loss $(\mathrm{AOR}=1.06, \mathrm{CI} 95 \%$ [1.03-1.09], $p<0.000)$.
Overall, the entire model containing all predictor variables in three blocks explained 23\% (Nagelkerke $R^{2}$ ) of the variance in worry about job loss, indicating that the model was able to distinguish between people with disabilities and chronic conditions who were worried to lose their jobs versus those who were not.

\section{Discussion}

This exploratory study aimed to examine whether higher levels of functional limitations among people with disabilities and chronic conditions were associated with greater worry about job loss during the COVID-19 pandemic. Our results revealed that the demographic variables of age, gender, and race were not associated with worry about job loss during the pandemic. COVID-19 related perceived stress was positively and significantly associated with worry about job loss. This finding is relatively consistent with previous research. For example, Baert et al. (2020) examined the expected impact of COVID19 on career outcomes and aspirations and found that about $21 \%$ of employees feared losing their jobs. Global Disability Inclusion (2020) examined the impact of COVID-19 on both individuals with and without disabilities. Results revealed that $13 \%$ of individuals with disabilities believed that they would lose their jobs in the next 1-3 months, which is about three times greater than what people without disabilities (5\%) reported (Global Disability Inclusion, 2020). In addition, $67 \%$ of individuals with disabilities reported that they would experience acute economic insecurity in the next 12 months, as compared to $52 \%$ of people without disabilities (Global Disability Inclusion, 2020). Indeed, with millions of Americans being out of work as a result of the pandemic, it would seem that the worry about job loss reported by participants in this study and in previous research is well justified.

The main research question in this study was whether functional limitations were significantly correlated with worry about job loss for individuals with disabilities and chronic conditions, after adjusting for demographic variables and perceived COVID19 stress. Not surprisingly, our results revealed that functional limitations were positively correlated with worry about job loss, even after controlling for demographic covariates and perceived COVID-19 stress. Vocational rehabilitation (VR) professionals working with people with the most significant disabilities 
during COVID-19 and in its aftermath must remain mindful that pre-existing severe disability effects not only make people more vulnerable to severe cases of COVID-19, they also pose potentially devastating threats to employability in our current time of unprecedented economic volatility and societal uncertainty.

People with disabilities, especially those with greater levels of functional limitations, may have experienced difficulties in accessing healthcare; transportation; and assistance with delivery of food, medication, and personal care support during this pandemic (Armitage \& Nellums, 2020; World Health Organization, 2020), which may make functional limitations even more problematic and consequently thwart their prospects for continued employment. Previous researchers have reported that there is a significant relationship between functioning and employment related outcomes in people with disabilities (Brucker et al., 2016; Lee et al., 2019; Muller et al., 2017; Vuong et al., 2015). Interestingly, previous research has not systematically examined the relationship between functional limitations and worry about job loss in individuals with disabilities; therefore, our findings contribute to the current literature by demonstrating the relationship between functional limitations and a precursor to job loss, namely, worry about being unemployed, among individuals with disabilities and chronic conditions during the COVID-19 pandemic.

Previous research has revealed that individuals with the most severe impairments and functional limitations were most likely to require accommodations to perform their jobs (Zwerling et al., 2003). Although the Americans with Disabilities Act (ADA) requires employers to provide reasonable accommodations to people with disabilities, due to economic challenges caused by COVID-19, employers may have difficulty in providing these accommodations. In addition, people with disabilities who are currently using accommodations at their workplaces may have difficulty using these accommodations to work remotely from home. Overall, difficulties with procuring on-the-job accommodations and shifting to remote or virtual work may be further increasing worry about losing jobs among individuals with disabilities who have higher functional limitations. This issue requires further inquiry.

Although not all people with disabilities are at high risk of contracting COVID-19 or experiencing more severe symptoms of the disease, there are some disability and chronic condition related factors (e.g., having underlying health problems) that may increase infection risk and more severe symptoms (Boyle et al., 2020). Jain-Link and Taylor Kennedy (2019) reported that only $39 \%$ of employed individuals with disabilities disclosed their disability to their managers, and only $21 \%$ of individuals with disabilities disclosed to human resources teams. The authors reported that "employees with disabilities who disclose [their disabilities] to most people they interact with are more than twice as likely to feel regularly happy or content at work than employees with disabilities who have not disclosed to anyone $(65 \%$ versus $27 \%$ ). They are also less likely to regularly feel nervous or anxious (18\% versus $40 \%$ ) or isolated (8\% versus 37\%)" (Jain-Link \& Taylor Kennedy (2019). During the pandemic, given that some people with disabilities may be more worried about their increased risk of contracting COVID-19 due to pre-existing health conditions, they may consider disclosing their disabilities to receive accommodations for remote working. However, disclosing a disability can be overwhelming as employees with disabilities may worry about how their employers might react to their disclosure and request for accommodations. Future research is needed in this area to examine whether receiving accommodations moderates the relationship between functional limitations and worry about job loss. In terms of clinical practice, rehabilitation counselors may discuss with their clients the need for accommodations and how to disclose them to employers. Rehabilitation counselors may also refer their clients to comprehensive accommodation resources such as the Job Accommodation Network (Rumrill et al., 2019) for assistance with COVID-19 related telecommuting and virtual workplace modifications.

People with disabilities experienced lower rates of employment than people without disabilities even prior to COVID-19. According to the Office of Disability Employment Policy (2020), the unemployment rate for people with disabilities (age of 16 and over) has increased from 7.2\% (August 2019) to $13.2 \%$ (August 2020). Compared to individuals without disabilities (23\%), 38\% of people with disabilities were laid off, furloughed or had to shut down their businesses due to the pandemic (Global Disability Inclusion, 2020). This could be partially due to difficulties in accessing accommodations to work remotely. Besides, people with disabilities are overrepresented in low-skilled occupations that are difficult to accommodate by working from home. In addition to accommodation factors, it is also impor- 
tant to consider the COVID-19 pandemic as a disaster, negatively affecting financial health in people with and without disabilities. For example, it was reported before the pandemic that people with disabilities disproportionately experienced unemployment and underemployment due to previous financial crises and the recession (Burke et al., 2013; Fogg et al., 2010). Therefore, it is important for us to examine protective and risk factors in employment and underemployment in people with disabilities during unexpected environmental crises such as the current pandemic. Our research revealed that, even after controlling for demographic variables and perceived COVID-19 stress, higher scores on functional limitations increased the probability of worrying about job loss among individuals with disabilities.

People with disabilities may face more challenges in finding and keeping their jobs due to personal and environmental factors (Anthony, 1994; Burke et al., 2013; Muller et al., 2017; Wang \& Lin, 2013). Given that COVID-19 increases the likelihood of unemployment and underemployment for both people with and without disabilities, rehabilitation counselors may further follow-up with their clients to understand specific needs to assist them in keeping their jobs during the pandemic, or in changing jobs if necessary. In research conducted by Global Disability Inclusion (2020) in April 2020, over 35\% of individuals with disabilities reported that they will need to gain new skills to find new employment. Although this finding needs to be further examined, rehabilitation counselors may help their clients who become unemployed due to COVID-19 to acquire new skills that will increase their employment options. Based on clients' needs and goals, rehabilitation counselors can also provide more tailored supports such as additional counseling services and benefits counseling services to help clients better manage their stress and anxiety and functional limitations during the pandemic. Rehabilitation counselors can also work with clients to explore triggers related to fear of losing their jobs, and identify and implement strategies to help clients gain an increased sense of self-esteem and manage their stress and anxiety.

\subsection{Limitations}

There are multiple limitations that VR professionals and researchers should consider when they interpret the results of this study. First, this study only consisted of a convenience sample of people with self-reported disabilities and chronic conditions.
Individuals who may be less comfortable navigating an online survey or may not have Internet access may have been left out of the study. Second, the sample was comprised of mainly whites and highly educated participants. Third, because worry about job loss was assessed using a single-item measure, a comprehensive scale may better capture the construct. In addition, we did not ask participants their specific jobs. This information could be helpful to examine the relationships among stress, functional limitations, and job loss worry among people who occupy highskilled versus low-skilled jobs. All of these factors may limit the generalizability of the study results to the broader population of people with disabilities.

\subsection{Conclusion}

Overall, our exploratory study contributes to the disability and vocational rehabilitation literature by examining the relationship between functional limitations and worry to lose jobs in people with disabilities. Results suggest that after controlling for demographic variables and stress, functional limitations were positively associated with worry about job loss amid the COVID-19 pandemic. Vocational rehabilitation professionals working with people with disabilities during and following COVID-19 must take into consideration specific disability-related work limitations and how those can be accommodated in consideration of social distancing requirements and the technological milieu of the new American workplace.

\section{Conflict of interest}

None to report.

\section{Funding}

This project was supported by the College of Health Sciences at the University of Texas at El Paso.

\section{References}

Anthony, W. A. (1994). Characteristics of people with psychiatric disabilities that are predictive of entry into the rehabilitation process and successful employment. Psychosocial Rehabilitation Journal, 17(3), 3-13. https://doi.org/10.1037/h0095578

Armitage, R., \& Nellums, L. B. (2020). The COVID-19 response must be disability inclusive. The Lancet Public Health, 5(5), e257. https://doi.org/10.1016/S2468-2667(20)30076-1 
Baert, S., Lippens, L., Moens, E., Sterkens, P., \& Weytjens, J. (2020). How do we think the COVID-19 crisis will affect our careers (if any remain)? (GLO Discussion Paper, Issue).

Boyle, C. A., Fox, M. H., Havercamp, S. M., \& Zubler, J. (2020). The public health response to the COVID-19 pandemic for people with disabilities. Disability and Health Journal, 13(3), 100943. https://doi.org/10.1016/j.dhjo.2020.100943

Brucker, D. L., Houtenville, A. J., \& Lauer, E. A. (2016). Using sensory, functional, and activity limitation data to estimate employment outcomes for working-age persons with disabilities in the United States. Journal of Disability Policy Studies, 27(3), 131-137.

Burke, J., Bezyak, J., Fraser, R. T., Pete, J., Ditchman, N., \& Chan, F. (2013). Employers' attitudes towards hiring and retaining people with disabilities: A Review of the literature. The Australian Journal of Rehabilitation Counselling, 19(1), 21-38. https://doi.org/10.1017/jrc.2013.2

Chan, F., Strauser, D., Maher, P., Lee, E.-J., Jones, R., \& Johnson, E. T. (2010). Demand-side factors related to employment of people with disabilities: A survey of employers in the Midwest Region of the United States. Journal of Occupational Rehabilitation, 20(4), 412-419. https://doi.org/ 10.1007/s10926-010-9252-6

Chiu, C.-Y., Chan, F., Bishop, M., da Silva Cardoso, E., \& O’Neill, J. (2013). State vocational rehabilitation services and employment in multiple sclerosis. Multiple Sclerosis Journal, 19(12), 1655-1664. https://doi.org/10.1177/1352458513482372

Dutta, A., Gervey, R., Chan, F., Chou, C.-C., \& Ditchman, N. (2008). Vocational rehabilitation services and employment outcomes for people with disabilities: A United States Study. Journal of Occupational Rehabilitation, 18(4), 326. https://doi.org/10.1007/s10926-008-9154-z

Fliege, H., Rose, M., Arck, P., Walter, O. B., Kocalevent, R.-D., Weber, C., \& Klapp, B. F. (2005). The Perceived Stress Questionnaire (PSQ) reconsidered: validation and reference values from different clinical and healthy adult samples. Psychosomatic Medicine, 67(1), 78-88

Fogg, N. P., Harrington, P. E., \& McMahon, B. T. (2010). The impact of the Great Recession upon the unemployment of Americans with disabilities. Journal of Vocational Rehabilitation, 33(3), 193-202.

Global Disability Inclusion. (2020). The Impact of COVID-19: People with Disabilities Compared to Those without Disabilities. Retrieved September 10, 2020 from https://www. globaldisabilityinclusion.com/download-whitepaper

Jain-Link, P., \& Taylor Kennedy, J. (2019). Why People Hide Their Disabilities at Work. Retrieved September 10, 2020 from https://hbr.org/2019/06/why-people-hide-their-di sabilities-at-work

Jalali, M., Shahabi, S., Bagheri Lankarani, K., Kamali, M., \& Mojgani, P. (2020). COVID-19 and disabled people: perspectives from Iran. Disability \& Society, 35(5), 844-847. https://doi.org/10.1080/09687599.2020.1754165

Kuper, H., \& Heydt, P. (2019). The mission billion: access to health services for 1 billion people with disabilities. Retrieved September 8, 2020 from https://www.lshtm.ac.uk/ TheMissingBillion

Lee, Y.-H., Chang, K.-H., Escorpizo, R., Chi, W.-C., Yen, C.-F., Liao, H.-F., Huang, S.-W., \& Liou, T.-H. (2019). Accuracy of the World Health Organization Disability Assessment Schedule 2.0 (WHODAS 2.0) score as an objective assessment tool for predicting return-to-work status after head and neck cancer in male survivors. Supportive Care in Cancer, 27(2), 433-441.

Lindesay, J., Baillon, S., Brugha, T., Dennis, M., Stewart, R., Araya, R., \& Meltzer, H. (2006). Worry content across the lifespan: an analysis of 16- to 74-year-old participants in the British National Survey of Psychiatric Morbidity 2000. Psychological Medicine, 36(11), 1625-1633. https://doi.org/ 10.1017/S0033291706008439

Muller, V., Chiu, C.-Y., Tang, X., Eagle, D., Peebles, M. C., Iwanaga, K., Brooks, J., \& Chan, F. (2017). Association of Employment and Health and Well-Being in People with Fibromyalgia. Journal of Rehabilitation, 83(3), 37-43.

Potts, B. (2005). Disability and employment: Considering the importance of social capital. Journal of Rehabilitation, 71(3), 20-25.

Rumrill, P., Hendricks, D.J., Elias, E., Jacobs, K., Leopold, A., Minton, D., Nardone, A., Sampson, E., Scherer, M., Stauffer, C., McMahon, B., Roessler, R., Rumrill, S., \& Taylor, A. (2019). An organizational case study of a five-year development project to promote cognitive support technology use, academic success, and competitive employment among civilian and veteran college students with traumatic brain injuries. Journal of Applied Rehabilitation Counseling, 50(1), 57-72.

Umucu, E., Iwanaga, K., Wu, J.-R., Brooks, J. M., Ditchman, N., Flowers-Benton, S., \& Chan, F. (2018). Preliminary Validation of a Short Form of the Perceived Stress Questionnaire for Use in Clinical Rehabilitation Counseling Research and Practice. Rehabilitation Research Policy and Education (4), 232-243. https://doi.org/10.1891/0889-7018.32.4.232

Umucu, E., \& Lee, B. (2020). Examining the impact of COVID-19 on stress and coping strategies in individuals with disabilities and chronic conditions. Rehabilitation Psychology, 65(3), 193198. https://doi.org/10.1037/rep0000328

Umucu, E., Tansey, T., Brooks, J., \& Lee, B. (2020). The protective role of character strengths in COVID-19 stress and well-being in individuals with chronic conditions and disabilities. Rehabilitation Counseling Bulletin.

Umucu, E., Villegas, D., Viramontes, R., Jung, H., \& Lee, B. (2020). Measuring grit in veterans with mental illnesses: Examining the model structure of grit. Psychiatric Rehabilitation Journal. Advance online publication. https:// doi.org/10.1037/prj0000420

U.S. Department of Labor Office of Disability Employment Policy (2020). Disability employment statistics. Retrieved from https://www.dol.gov/agencies/odep/publications/statistics.

Üstün, T. B., Chatterji, S., Kostanjsek, N., Rehm, J., Kennedy, C., Epping-Jordan, J., Saxena, S., Korff, M. v., \& Pull, C. (2010). Developing the World Health Organization disability assessment schedule 2.0. Bulletin of the World Health Organization, $88,815-823$.

Vuong, T. D., Wei, F., \& Beverly, C. J. (2015). Absenteeism due to Functional Limitations Caused by Seven Common Chronic Diseases in US Workers. Journal of occupational and environmental medicine, 57(7), 779-784. https:// doi.org/10.1097/JOM.0000000000000452

Wang, Y.-T., \& Lin, Y.-J. (2013). Employment Outcome Predictors for People with Disabilities in Taiwan -A Preliminary Study Using ICF Conceptual Frameworks. Journal of Rehabilitation, 79(2), 3-14.

World Health Organization. (2020). Disability considerations during the COVID-19 outbreak. World Health Organization. Retrieved September 8, 2020 from https://apps.who. 
int/iris/bitstream/handle/10665/332015/WHO-2019-nCovDisability-2020.1-eng.pdf

Zwerling, C., Whitten, P. S., Sprince, N. L., Davis, C. S., Wallace, R. B., Jd, P. B., \& Heeringa, S. G. (2003). Workplace Accommodations for People with Disabilities: National Health
Interview Survey Disability Supplement, 1994-1995. Journal of Occupational and Environmental Medicine, 45(5). https:// journals.lww.com/joem/Fulltext/2003/05000/Workplace_

Accommodations_for_People_with.12.aspx 